Dirección

Clara Martínez Cantón

Gimena del Rio Riande Ernesto Priani

Secretaría Romina De León

RHD 3 (2019)

\section{Seminario "Historia y Humanidades Digitales". Ciudad Real, 7-9 de} noviembre de 2017.

Durante los días 7,8 y 9 de noviembre de 2017 se celebró en la Facultad de Letras del Campus Universitario de Ciudad Real un seminario destinado a presentar algunas cuestiones relacionadas con la importancia con la que la tecnología ha calado también en el ámbito académico y la investigación histórica. Son innumerables las ventajas tecnológicas con las que hoy en día cuenta el trabajo histórico, facilitando tanto la investigación como la labor docente. El seminario consistió en un ciclo de conferencias, un taller y una exposición y estuvo organizado por los profesores David Martín López y Francisco J. Moreno Díaz del Campo, de la Universidad de Castilla-La Mancha, y Francisco Fernández Izquierdo, del Consejo Superior de Investigaciones Científicas (CSIC), siendo esta actividad una muestra más de la colaboración entre estas instituciones.

El ciclo de conferencias se inició con la intervención titulada "Integración de bases de datos y visualización, una experiencia interdisciplinar en el proyecto ForSEAdiscovery" a cargo de Ana Crespo, del Instituto de Historia del CSIC. Este proyecto ha sido financiado por el programa europeo Marie Curie y participan en él investigadores de distintos países, universidades y centros de investigación. El proyecto trata de integrar datos históricos con información geográfica en bases de datos compartidos a través de distintas bases y modelos de datos como Sistemas de Informacion Geográfica (SIG). Los objetivos que este proyecto busca alcanzar son la consolidación de una línea de investigación que combine investigación histórica, arqueología subacuática, SIG y métodos de procedencia de madera como dendrocronología, anatomía de la madera y geo-dendroquímica. 
El aumento de los antecedentes y la experiencia de los aprendices en las diferentes áreas de investigación mediante la participación de los becarios en cursos de capacitación y talleres destinados a desarrollar sus habilidades científicas, de comunicación y de gestión; y el desarrollo de habilidades para futuras carreras en la academia o el sector privado mientras se avanza en los campos de investigación mediante la integración de herramientas de investigación, el desarrollo de conjuntos de datos de referencia y nuevos descubrimientos. La estructuración del proyecto de investigación se lleva a cabo a través de paquetes de datos y se pretende divulgar los conocimientos obtenidos en la sociedad.

La siguiente ponencia corrió a manos de José María Imízcoz, de la Universidad del País Vasco. Esta conferencia se centró en la perspectiva de la investigación, no de la divulgación y cómo a través del análisis de las redes sociales podemos crear una historia conectada. Para ello debemos partir de la idea de que los hombres y las mujeres en sociedad somos actores de nuestra historia política, económica, social, y cultural, por tanto, el objeto de las investigaciones siempre deben ser las agrupaciones y la colectivización para poder percibir sus relaciones con el contexto. Este análisis relacional emplea diversas técnicas que pueden utilizarse solas o combinadas como la microhistoria, la biografía, la prosopografía, el análisis de redes sociales, etc. Más allá de las técnicas empleadas, tienen en común el procedimiento inductivo. El análisis de redes sociales es un método que investiga el conjunto de interacciones entre los individuos que interactúan en un momento dado para captar formas complejas de interacción social como las conexiones sociales, políticas, familiares. También para comprender cómo interactúan los comportamientos individuales con el entorno general. La mayor densidad de redes sociales tiene que ver con una tendencia de un sector social a la reproducción de patrones de comportamiento y actuación o por el contrario, al cambio. Hay que tener en cuenta la existencia de los llamados lazos débiles que perjudican a ciertos individuos haciendo que solo accedan a noticias limitadas y a las opiniones de sus íntimos, viéndose privados de la información que procede de partes distantes del sistema social. A escala general las sociedades que carecen de lazos comunicativos firmes están fragmentadas, las ideas se difunden lentamente y los subgrupos tienen dificultades para articularse entre sí. Otra forma de análisis sería la prosopografía relacional, que estudia un colectivo no con los rasgos genéricos, sino mediante el conocimiento detallado del conjunto de los componentes, individualmente. Existen distintas bases de datos destinadas a almacenar fichas con esta información, siendo Fichoz la más conocida.

La segunda sesión de conferencias se inició con la intervención del profesor de la Universidad de Murcia, Juan Francisco Jiménez, bajo el título "Yo estuve allí. La experiencia de la Historia vivida a través de un videojuego". Cuando hay que definir lo que es un videojuego, nos encontramos con respuestas de todo tipo. En cualquier caso podemos afirmar que los videojuegos forman parte de la cultura y el aprendizaje, aunque el mundo académico en su mayoría no lo acepte, ya que hay muchos prejuicios en torno a ellos. Cabe afirmar que estamos 
viviendo un cambio general en la civilización, los videojuegos son una consecuencia más, derivada del surgimiento de una nueva cultura. Los videojuegos pueden ayudar a los profesionales de la Historia a desarrollar la percepción del tiempo ya que nos conducen a hacernos preguntas como żdónde y cómo aprendo la historia?, ¿̇quién me la cuenta?, ¿̇dónde la veo? Hasta ahora el pasado podíamos escucharlo a través de la Historia Oral y podíamos leerlo, pero actualmente gracias al desarrollo de los videojuegos también podemos vivir la Historia, ya que nos podemos sumergir directamente en momentos históricos precisos. Además, la mayoría de ellos poseen un carácter inmersivo donde es el jugador el que decide activamente el resultado final. Acontecimientos históricos como la Revolución Francesa, la América Colonial o la Gran Guerra cobran vida cuando el usuario, en lugar de estudiarlos o leer libros que, a menudo contienen mares de tecnicismos difícilmente comprensibles por la población inexperta, los encarna en primera persona, apreciando con gran detalle y con especial cercanía aspectos que -frecuentemente- se pasan por alto si tratan de interiorizarse sin conocimientos muy específicos o que pueden resultar tediosos en los medios tradicionales. En síntesis, el uso del videojuego en el aula es una revolución en el proceso de aprendizaje y enseñanza de la Historia.

A continuación, el profesor Francisco Alía Miranda, de la Universidad de Castilla-La Mancha, presentó la conferencia "Recursos digitales para la investigación histórica". En la red pueden encontrarse diversas plataformas que pueden ayudarnos en nuestra tarea de investigación. En primer lugar encontramos las bases de datos, que son conjuntos de datos de carácter homogéneo y que se refieren a una materia $\circ$ problema determinado, son legibles desde un ordenador y pueden ser bibliografías y catálogos. Algunos ejemplos de bases de datos a las que podemos acceder a través de internet son Ceres, Dialnet, Pares, Periodicals Archive Online y Worldcat. En la red también podemos encontrar diversas colecciones documentales y bibliográficas recopiladas por distintas instituciones como la Fundación histórica Tavera con su Biblioteca digital clásicos Tavera y el Instituto Nacional de Estadística con sus publicaciones históricas en soporte electrónico como el Censo de Pecheros. En cuanto a bibliotecas y archivos digitales podemos destacar American History, el Archivo virtual de la Edad de Plata de la cultura española contemporánea (1868-1936), la Biblioteca Digital de Castilla-La Mancha, la Biblioteca Digital de la Real Academia de la Historia y la Biblioteca Virtual del Patrimonio Bibliográfico. A través de nuestros dispositivos digitales también podemos acceder a determinados repositorios y recolectores. Un repositorio es un espacio virtual donde se almacena y se mantiene la documentación digital, son depósitos de información sin organización por lo que no hay que confundirlos con una biblioteca digital. Por su parte, un recolector es un servidor externo fuera de los repositorios y las bibliotecas digitales que permite que el usuario localice de manera simultánea la información depositada en algunos de ellos. Algunos ejemplos de repositorios y recolectores son Digital CSIC, Hispana y Recolecta. Las hemerotecas virtuales 
como la Hemeroteca $A B C$, la Hemeroteca de La Vanguardia, o la Hemeroteca de la Biblioteca Virtual Miguel de Cervantes. Por último tenemos fototecas y filmotecas en red como $A B C$ foto, Filmoteca Española Online de RTVE y la Fototeca del Patrimonio Histórico.

El ciclo de conferencias se cerró el día 9 de noviembre con las intervenciones de Anaclet Pons, de la Universidad de Valencia, y Antonio Rodríguez de las Heras, de la Universidad Carlos III. El primero expuso en "La Historia digital" cómo en los últimos tiempos debido al desarrollo de la tecnología, ha cambiado la manera en la que generamos los documentos, registramos la experiencia y la comunicamos. También se ha producido una variación en la forma de escribir la información. Cuando se modifica la manera de pensar el mundo que nos rodea, cambia también la manera de percibir el pasado. Si a día de hoy triunfa la Historia de las emociones es porque el punto de mira está puesto en las emociones y las aplicamos al pasado. Según el profesor Pons existen dos planos a la hora de definir qué son la Historia y las Humanidades Digitales. Por un lado, están las Humanidades y la Historia en la era digital, y por otra, éstas mismas en carácter digital. La primera, es el hecho de que los medios con los que producimos han cambiado. La segunda, es otro plano que ha recibido muchas críticas, pues hacer Historia digital implica hacer una apuesta epistemológica porque se quiere hacer algo distinto con las herramientas digitales. Tanto en un caso como en otro hay un problema que supera a estos dos planos: hay un cambio general que nos afecta a todos por igual y es independiente de que vivamos o no en el mundo digital.

La desmaterialización de la información tiene como consecuencia un cambio de sistema literario moderno, es decir, en la creación literaria. Se ha producido un cambio en el concepto de autor, en la creación y en el mercado literario. Todo lo que se construyó durante décadas alrededor del mundo impreso se encuentra actualmente en crisis o mutación debido a la aparición de nuevas tecnologías. Un ejemplo de cambio de soporte que ha implicado modificaciones en la forma de investigar y difundir la información fue la aparición de la máquina de escribir. La adquisición de esta herramienta produjo que el pensamiento sistemático y tradicional de Nietzsche diese paso a un pensamiento aforístico, en el que la manera en la que se escribe determina el pensamiento. Tras esto, cuando aparecen el gramófono y el cinematógrafo se aúnan dos canales, imagen y sonido, que a su vez se separan de la palabra impresa. Esto provocó un cambio en la literatura de la época, pues la escritura perdió el poder alucinatorio que tenía anteriormente. El mundo derivado de las palabras, murió. Con la aparición de este tipo de tecnologías ya no se tenía el monopolio alfabético para impresionar y describir, sino que la imagen y el sonido poseen una implicación más directa.

El ciclo de conferencias se cerró con la intervención del mencionado Rodríguez de las Heras, bajo el título "Humanidades y la educación en red". Actualmente estamos viviendo en un tiempo en el que se desarrolla a gran velocidad la cultura digital. La educación en la red ya es una realidad, todo gracias a la evolución de los medios y al desarrollo de Internet. Dicha cultura 
digital ha resultado del desarrollo parejo que han tomado la tecnología y las humanidades, pero hasta que todo el mundo no pueda acceder a los recursos tecnológicos no se desarrollará correctamente. Hay que tener muy en cuenta que la educación en red no es educación a distancia. Hay tres sistemas educativos: presencial, a distancia y en red. Esta última se produce por una serie de factores. Hay que entender la tecnología como una prótesis: funciona bien cuando no la sientes, por tanto, tenemos que normalizar los usos tecnológicos en nuestra vida, incluyendo en el ámbito educativo y del aprendizaje. Con respecto a esta cuestión, el profesor Rodríguez de las Heras señaló que frente a los cursos MOOC (Massive Online Open Course) que están basados en vídeos principalmente, se está llevando a la práctica algo similar pero que basado en audios que involucran al alumno en una especie de conversación con el profesor ya que resultan mucho menos artificiales que los vídeos y por tanto el aprendizaje es más intenso.

La segunda actividad que se desarrolló en el seminario fue un taller de introducción al uso de los gestores de bibliografía, que sirvió para enseñar a los alumnos una potente herramienta de cara a la búsqueda, localización y uso de bibliografía en la preparación de textos académicos. Fue impartido por el mencionado Francisco Fernández Izquierdo y estuvo dividido en tres sesiones, teniendo lugar en la segunda parte de cada una de las tardes que duró el seminario.

Durante la sesión de introducción, se explicó la utilidad de Zotero, un gestor bibliográfico desarrollado por el Center for History and New Media de la Universidad George Manson. Instalamos el programa en nuestros ordenadores. Se habló de la importancia de trabajar con este tipo de gestores que permiten tener acceso a toda la información bibliográfica sobre los documentos con los que los profesionales de la Historia trabajamos. Se detallaron los usos más básicos de Zotero y cómo introducir en el programa informaciones bibliográficas extrayéndolas de diversos portales en red como Dialnet. Se dio mucha importancia a que las citas bibliográficas obtenidas pueden aparecer en distintos lenguajes informáticos que deben ser comprendidos, ya que hay algunos que el programa no es capaz de interpretar.

A lo largo de las tardes se fueron ampliando los conocimientos de base anteriormente detallados, además de aprender nuevas herramientas que pueden utilizarse en Zotero, como la creación de carpetas para organizar mejor la información, borrar determinados ficheros duplicados y cambiar algunos datos de las fichas bibliográficas que por defecto se guardan de forma errónea o con falta de algunos datos.

Para terminar, la tercera actividad consistió en la exposición "Videojuegos y Edad Media", que pudo visitarse en el hall de entrada de la Facultad de Letras y que fue cedida por 
el grupo de investigación del mencionado Juan Francisco Jiménez¹. Como señaló en su ponencia, las últimas tres décadas han supuesto una revolución cultural sin precedentes, en la que el desarrollo tecnológico ha servido de perfecto hilo conductor para la implosión del fenómeno audiovisual. Industrias como la cinematográfica $y$, especialmente, la del videojuego han alcanzado unas cotas de éxito y popularidad nunca antes vistas. En los distintos paneles de la muestra se hace hincapié en la tesis que defendió el profesor Jiménez Alcázar, relativa al potente recurso didáctico en que se han convertido los videojuegos, debido a toda la información que ofrecen (cada vez más) y a la eficacia del aprendizaje inmersivo que se produce en ellos. Todo ello, fijándose expresamente en cómo conocer el medievo a través de juegos como Assasin's Credd. Syndicate, Medieval Total War II ○ Age of Empires II: The African Kingdoms, entre otros.

A modo de conclusión, podemos decir que poco a poco la tecnología ha incrementado de forma positiva el proceso de aprendizaje del alumnado, puesto que son muchos los beneficios que tiene en el ámbito educativo. El mundo docente debe adecuarse a las circunstancias con las que la sociedad progresa y, por tanto, la aplicación de la tecnología, algo tan corriente y necesario ya en todos los ámbitos de nuestra vida, debe llegar también a las aulas. Esta tiene un papel vital en la formación del alumnado, actuando como vehículo conductor de la información y teniendo un carácter directo y eficiente. En el seminario se desarrollaron todas estas cuestiones, además de enseñar qué utilidad tiene el uso de la tecnología en el ámbito específico de la Historia. Para el trabajo de los profesionales de la Historia es muy necesario el manejo de las fuentes de información, y con las nuevas tecnologías se puede acceder a estas más fácilmente, analizarla, y almacenarla.

1 Accesible desde: www.historiayvideojuegos.com. 